his book is essentially a collection of the results which he and his co-workers have obtained by the application of scaling ideas. These results have been largely confirmed by the new experimental methods and the general agreement between theory and experiment is most impressive. However, the scaling method only gives an understanding of 'universal' properties such as, for example, the molecular weight dependence of the dimensions of a flexible polymer molecule in solution. Numerical prefactors, which are specific to a given monomer, are not predicted by this approach.
In the book the scaling concept is applied to a great variety of topics. Polymers in dilute and semi-dilute solution, theta solvents and good solvents, gels, melts and the dynamics of single chains and networks are just some of the problems successfully treated. It is not easy to apply scaling ideas and much physical insight is needed to get correct results. De Gennes has such insight and he is also adept at explaining his reasoning. Further work is now needed to derive scaling results in a more systematic way, and in the last chapter de Gennes outlines the renormalization group which probably provides the best way of putting the scaling approach on a firmer theoretical foundation.

The book is written mainly for experimentalists in polymer sciences who wish to incorporate the recent theoretical advances into their mode of thinking and for polymer theorists who want to achieve a qualitative understanding of the scaling method. It is an excellent work and no worker in the field can afford to be without a copy.

M. A. Moore is Professor of Theoretical Physics in the University of Manchester, UK

\section{Applied bifurcation theory}

\section{Michael Berry}

Bifurcation Theory and Applications in Scientific Disciplines. Edited by O. Gurel and O.E. Rössler. Pp. 708. (New York Academy of Sciences: New York, 1979.) $\$ 85$.

THIS is a collection of over 50 papers presented at a conference held in New York in 1977. Bifurcation is sudden qualitative change in the solutions of an equation or system of equations, produced by the smooth variation of one or more parameters. As might be expected on the basis of such a general definition the papers cover a vast range of subjects, well indicated by the titles of sections into which the book is divided: mathematics, biology, chemistry and chemical engineering, physics, ecology, economics, engineering, and experimentation and simulation. What all these subjects have in common is that they involve nonlinear equations, and the reason for the emergence of bifurcation theory as a separate discipline is that for some classes of nonlinear equations the bifurcation behaviour is beginning to be well understood.

\section{Source book on cadmium}

\section{M.H. Martin}

The Chemistry, Biochemistry and Biology of Cadmium. Edited by M. Webb. Pp.476. (Elsevier/North-Holland: Amsterdam and New York, 1980.) Dfl. 165, $\$ 80.50$.

THIS book is the second volume in a series on topics in environmental health, and consists of 11 chapters by different authors.

Most of the contributions are concerned with mammals including human beings, and indeed the editor states that the book attempts to integrate the toxicological aspects of cadmium in animals (sensu mammals) and human beings with the chemistry and biochemistry of the cadmium ion. The singling out of soils, plants and aquatic organisms as other environmental components to be considered serves to highlight the absence

Blackwell Scientific

In their advertisement (p.xxi) in the 12th June issue of Nature, titles and authors of two books were transposed. Introduction to Modern Virology, 2nd edn, is by S.B. Primrose and N.J. Dimmock; Pharmaceutical Microbiology, 2nd edn, is by W. B. Hugo and A.D. Russell. of contributions on air, terrestrial invertebrates and non-mammalian terrestrial vertebrates. In addition the preponderance of discussion on metallothioneins (cadmium, zinc, copper and mercury), some three chapters amounting to $22 \%$ of the book and also mentioned in the aquatic context, further unbalances the presentation.

In his remarkably brief preface, scarcely half a page, the editor states that he expects that the individual chapters of the book will be read as complete entities. To this end the various articles provide useful reviews on specific topics by authoritative contributors. The various chapters are relatively up to date, most including references to some 1979 literature, although some appear to have been completed before others. In fairness to the individual authors space could have been found in the preface to mention dates of completion of the reviews.

The presentation is relatively lavish and is consistent with the Elsevier/North Holland Biomedical Press standards, with matching price. Like many books of this multi-author type, its main use is likely to be as a source book of specific aspects of cadmium biochemistry rather than as a comprehensive treatment of the environmental biology, toxicology and biochemistry of cadmium.

M. H. Martin is Senior Lecturer in Botany at the University of Bristol, $U K$.
In the simplest case of all, dynamics is ignored (i.e. the system's state is considered to change very rapidly) and bifurcation theory reduces to the classification of equilibria, accomplished by Thom's theory of catastrophes. In several papers here a refined version is applied to analyse the types of buckling that occur in elastic structures under increasing load in the presence of imperfection (of design or manufacture) described by several parameters.

When dynamics is taken into account, the variety of possible bifurcations is much greater, but the following sequence of events is quite common: as a parameter $P$ increases, a stable equilibrium bifurcates into a stable closed orbit (Hopf bifurcation), which in turn bifurcates into one of twice the length, and so on, in a cascade of successive 'subharmonic bifurcations' accumulating at a finite value of $P$, beyond which the motion is aperiodic and chaotic. Such behaviour occurs, for example, in the ecological equations for the numbers of individuals in successive generations of a population reproducing in an environment with finite resources, or in the physiological control equations governing respiratory and haematological disorders, or in preturbulent fluid regimes with different Reynolds' numbers. In several papers the mathematical models give a very good description of experimental results, but when structurally stable behaviour is expected (and found) in a wide variety of contexts, it is important to be quite precise about what theory is being tested.

The general standard of exposition is high, but I cannot resist quoting one example of intolerable jargon:

The bootstrap principle of adaptability theory is: The assumption of the hierarchical and compartmental structurability of the state description of biological systems is self-justifying within the framework of adaptability theory in the scnse that such structurability is a necessary condition for efficient adaptability.

I strongly recommend this book to anybody wanting an introduction to this fertile new field of applied mathematics. [

Michael Berry is Professor of Physics at the University of Bristol, UK. 